\title{
Effect of aspirin on intestinal absorption of glucose, sodium, and water in man
}

\author{
C. ARVANitAKIS ${ }^{1}$, G-H. CHEN, J. FOLSCROFT, AND N. J. GREENBERGER \\ From the Department of Medicine, Division of Gastroenterology, Kansas University School of Medicine, \\ Kansas City, Kansas, USA
}

SUMMARY The effect of aspirin on small intestinal function in six healthy volunteers was examined using a segmental perfusion technique, with a test solution of $40 \mathrm{mM} \mathrm{D}$-glucose, $140 \mathrm{mM} \mathrm{NaCl}$, and $0.5 \%$ polyethylene glycol. Jejunal glucose, sodium, and water absorption rates were inhibited by $50 \%$ after oral administration of $2.6 \mathrm{~g}$ aspirin. Adenosine triphosphate (ATP) concentration was assayed in jejunal mucosal biopsies before and after aspirin. There was an almost $50 \%$ decrease in mucosal ATP levels after aspirin. This effect may be mediated through cellular injury and impairment of mitochondrial energy metabolism. These data suggest that aspirin may significantly alter small intestinal function. It appears possible that the inhibitory effect of aspirin on glucose absorption may account, at least in part, for the lower blood sugar levels observed with the use of the drug.

Aspirin is one of the most widely used drugs with diverse pharmacological and metabolic effects on a variety of biological systems. The effects of salicylates on carbohydrate metabolism are complex and not well understood. Previous studies have shown that salicylates in therapeutic doses may influence glucose homeostasis by lowering blood glucose in normal and diabetic subjects (Hecht and Goldner, 1959; Reid and Lightbody, 1959; Gilgore, 1960).

Despite numerous experimental studies, the mechanism of the hypoglycaemic action of aspirin has not been established. It is likely that the effect of salicylates on blood glucose is not mediated by stimulation of insulin release or enhanced sensitivity to circulating insulin, as it is demonstrable in the alloxan-diabetic rat (Smith et al., 1952). These authors have shown that salicylates reduced glucosuria and blood glucose in the diabetic rat, but there was no change in the liver-glycogen content. On the other hand, salicylates may inhibit gluconeogenesis from various metabolic intermediaries (Gould and Smith, 1965), possibly because of decreased availability of ATP, a necessary requirement for metabolic processes which are energy-dependent.

As glucose transport is an energy-dependent process against an electrochemical gradient and glucose

1Address for correspondence: Dr Constantine Arvanitakis,
Department of Medicine, Kansas University Medical Center,
Rainbow Blvd. at 39th Street, Kansas City, Kansas 66103,
USA.

Received for publication 6 October 1976 homeostasis is influenced by the rate of intestinal glucose absorption, it may be asked whether salicylates affect glucose levels by impairing glucose transport. Although the effect of aspirin on gastric mucosal structure and function has been studied extensively, there is very little information regarding the effect of aspirin on small intestinal function. Aspirin is absorbed from the stomach, as well as from the mucosa of the upper small intestine. The rate of absorption depends on the amount of nonionised drug and its lipid solubility. Because of the low intragastric $\mathrm{pH}$, aspirin is absorbed rapidly from the gastric mucosa in the nonionised form. However, an appreciable amount is also absorbed from the jejunum, since the intraluminal $\mathrm{pH}$ in proximal

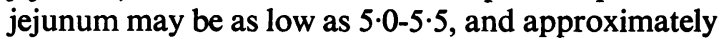
$10 \%$ aspirin would be still in a nonionised form, suitable for absorption (Woodbury, 1970). The large jejunal absorptive surface and the intestinal blood flow are also important factors which would influence aspirin absorption rates. Contact of aspirin with the brush border may have an effect on intestinal transport of nutrients predominantly absorbed from the jejunum.

The purpose of this study was to examine the effect of a therapeutic dose of aspirin on jejunal absorption of glucose, sodium, and water. In addition, the effect of aspirin on ATP levels in jejunal biopsies was determined, since glucose and sodium transport depend on high energy compounds such as ATP. 
Methods

Six normal subjects (four males and two females), ranging in age from 22-45 years, took part in the studies. None of the subjects had a history of diabetes, gastrointestinal, pancreatic, or liver disease, or was taking salicylates on a regular basis. Informed consent was obtained from all the subjects before the study. The perfusion studies were performed at the Clinical Research Center of the Kansas University Medical Center. The protocol for these studies was approved by the Human Experimentation Committee of the Kansas University College of Health Sciences. After an overnight fast, the proximal jejunum was intubated with a triple lumen tube and the position of the tube was determined fluoroscopically. The infusion site of the tube was at the area of the ligament of Treitz, with aspirating sites 15 and $45 \mathrm{~cm}$ distal to the infusion opening in the jejunum. Jejunal perfusion studies were performed (Cooper et al., 1966) with an isotonic test solution (osmolality $292 \pm 12 \mathrm{mOsm} / \mathrm{l}$, mean $\pm \mathrm{SD}$ ), consisting of $40 \mathrm{mmol} / 1 \mathrm{D}$-glucose, $140 \mathrm{mmol} / \mathrm{l} \mathrm{NaCl}$, and $0.5 \%$ polyethylene glycol (PEG 4000 ), as the non-absorbable marker, at a constant rate of $14 \mathrm{ml} /$ min (Harvard Apparatus, Mills, Massachusetts). After a 30-minute equilibration period, three 20minute collections were obtained from the proximal and distal aspirating sites. The perfusion was then stopped and $2.6 \mathrm{~g}$ aspirin were given by mouth in $1 \%$ methylcellulose suspension. After 30 minutes, to allow absorption of aspirin, the perfusion was started again, and after another 30-minute equilibration period, three more 20-minute collections were made.

Blood was drawn fasting, at the end of the control period, and after aspirin administration, for serum immunoreactive insulin (Morgan et al., 1964) and glucose determination (Huggett and Nixon, 1957). Serum salicylic levels were determined at 0 , one, and two hours after aspirin ingestion (Trinder, 1954). Duplicate aliquots of perfusion solution, and proximal and distal collections, were deproteinised (Somogyi, 1945) and the filtrate was assayed for glucose (Huggett and Nixon, 1957), polyethylene glycol (Malawer and Powell, 1967), and sodium by flame photometry. Net absorption rates of glucose, water, and sodium were calculated using standard formulas (Whalen et al., 1966).

In separate experiments, jejunal biopsies were obtained from the area at the ligament of Treitz (Brandborg et al., 1959), from four normal volunteers, before and one hour after ingestion of $2.6 \mathrm{~g}$ aspirin in $1 \%$ methylcellulose suspension. Tissue was collected immediately in boiling water and adenosine triphosphate (ATP) concentrations were determined in the tissue extract using firefly extract (Firefly Luciferase, Worthington Biochemical Corp.). ATP was determined by measuring luminescence with a photomultiplier Farrand photofluorometer (Strehler and Totter, 1954). Results were expressed in $\mu \mathrm{mol}$ ATP/mg wet tissue weight. Student's $t$ test (paired data) was used for statistical analysis of the results.

Control perfusions were done in two additional studies.

\section{Results}

Aspirin administration resulted in a marked decrease of net jejunal absorption of glucose, sodium, and water (Fig. 1). Mean glucose absorption decreased from $14.5 \pm 6(1 \mathrm{SD}) \mathrm{mmol} / \mathrm{h} / \mathrm{segment}$ to $8 \cdot 7 \pm 4$ (1 SD) $(\mathrm{P}<0.05)$, sodium absorption from $31 \pm 14$ (1 SD) $\mathrm{mEq} / \mathrm{h} / \mathrm{segment}$ to $14.0 \pm 8(1 \mathrm{SD})(\mathrm{P}<$ $0.025)$, and water absorption from $261 \pm 122$ (1 SD) $\mathrm{ml} / \mathrm{h} /$ segment to $135 \pm 52$ (1 SD) $(\mathrm{P}<0.05)$. ATP concentration in the jejunal biopsies was similarly decreased after aspirin administration from 12.03 \pm 5 (1 SD) $\mu \mathrm{mol} / \mathrm{mg}$ wet tissue weight to $6.3 \pm 3.5$ (1 SD) $(P<0.05)$ (Fig. 2). The results of blood sugar, insulin, and serum salicylic levels are summarised in the Table. There was no statistically significant difference in blood glucose determination and insulin levels. Salicylate levels were $0.54 \pm 0.11$ and $0.56 \pm$ $0 \cdot 10 \mathrm{mmol} / 1$ (mean $\pm \mathrm{SD}$ ) at one and two hours respectively after aspirin ingestion. These are considered to be low therapeutic levels, but a larger dose of aspirin, which might have resulted in higher levels, was avoided to prevent potential side-effects.

\section{Discussion}

The results of this study indicate that aspirin administration caused a marked depression of glucose, sodium, and water jejunal absorption in man. Jejunal mucosal concentration of ATP was similarly decreased after aspirin by approximately $50 \%$. A possible explanation for the inhibitory effect of aspirin on intestinal absorption of nutrients which require energy compounds is that the drug may affect mitochondrial metabolism. The decreased availability of ATP would furthersupport the possibility of alteration in mitochondrial function. It is of interest that gastric mucosal ATP levels were irreversibly reduced in isolated preparations of the bullfrog stomach by mucosal instillation of salicylic acid (Kasbekar, 1973). Similarly, gastric mucosal ATP content was found to be reduced after mucosal instillation of aspirin in the canine stomach (Kuo and Shanbour, 1976). A reduction in ATP concentration by aspirin may be related to the uncoupling and inhibitory effect of the drug on oxidative phosphorylation 


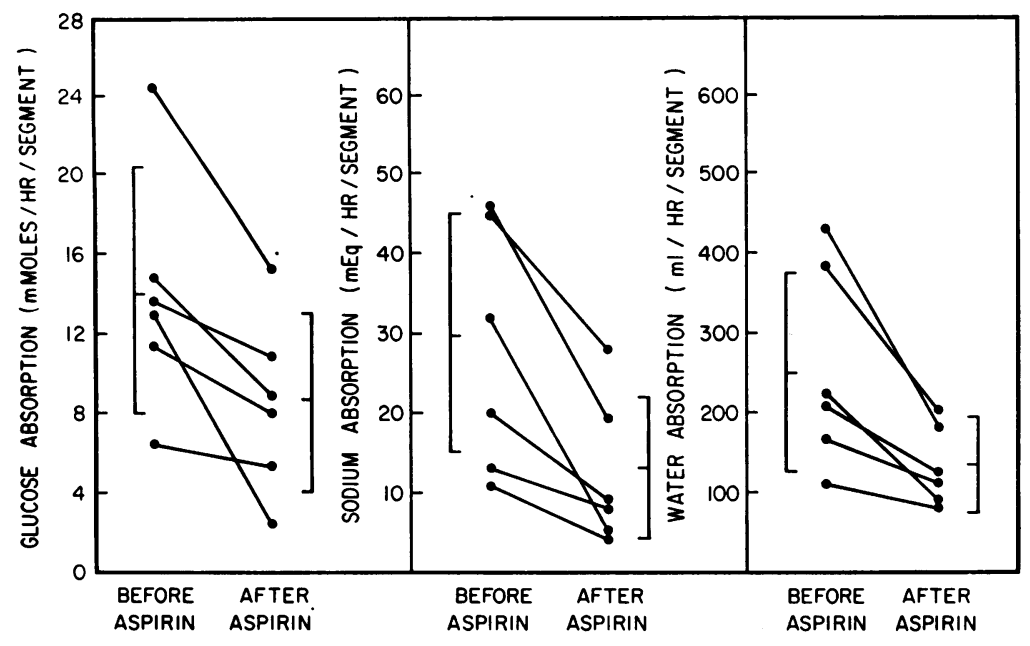

Fig. 1 Glucose, sodium, and water absorption before and after aspirin administration. Mean $\pm S D$ is shown in each column. A statistically significant decrease in the net absorption of glucose, sodium, and water was observed after aspirin.

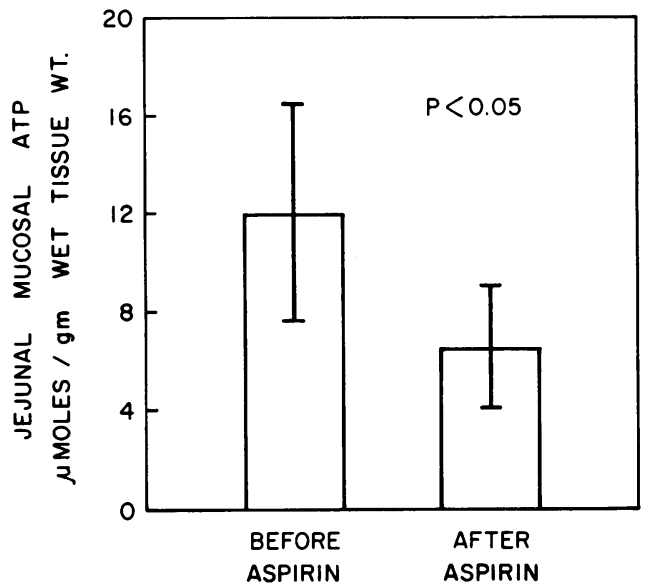

Fig. 2 Jejunal mucosal ATP concentration before and after aspirin (mean $\pm S D$ ). There was a $50 \%$ reduction in ATP after aspirin.

(Brody, 1955; Pachman et al., 1971). Such uncoupling and inhibitory effect of the drug on oxidative phosphorylation (Brody, 1955; Pachman et al., 1971). Such uncoupling would cause a decrease in cyclic AMP levels by reducing the available concen-

Table Blood glucose, insulin (IRI) and serum salicylic acid levels before and after aspirin administration $($ mean $+S D)$

\begin{tabular}{lccc}
\hline & Before aspirin & After aspirin & $P$ \\
\hline Blood glucose $(\mathrm{mmol} / \mathrm{l})$ & $5.11 \pm 0.66$ & $4.67 \pm 0.61$ & N.S. \\
Insulin (IRI) $(\mu \mathrm{U} / \mathrm{ml})$ & $12.3 \pm 7$ & $16.7 \pm 8$ & N.S. \\
Serum salicylic acid (mmol/l) & & & \\
$1 \mathrm{~h}$ & - & $0.54 \pm 0.11$ & \\
$2 \mathrm{~h}$ & - & $0.56 \pm 0.10$ & \\
\hline
\end{tabular}

tration of ATP as a substrate for adenyl cyclase. In fact, it has been shown that oral administration of large doses of aspirin (10-15 g) in normal volunteers resulted in significant reduction of cyclic AMP levels in lymphocytes (Snider and Parker, 1975).

It is reasonable to assume that intestinal mitochondria may be vulnerable to an orally administered agent, especially when there is evidence of deleterious effects on cell systems dependent on mitochondrial function. It will be of interest to correlate the functional changes of intestinal absorption noted in our study with possible structural alterations of intestinal epithelial cells. In one study, Hahn et al. (1975) examined the effect of aspirin on the ultrastructure of jejunal mucosa after administration of $\mathbf{3} \mathbf{g}$ aspirin daily for three to five days. They found a marked increase of secondary lysosomes, probably representing autophagolysosomes, as well as an increase of multivesicular bodies. These morphological changes indicate that intracellular concentration of aspirin in intestinal epithelial cells during absorption may cause cellular injury and interfere with metabolic processes.

In conclusion, aspirin in a therapeutic dose produced an inhibitory effect on glucose, sodium, and water absorption in man. It is possible that this effect is not specific for glucose transport, but rather related to alteration of energy-dependent processes. Although the design of the study did not determine a hypoglycaemic effect, it is possible that the influence of aspirin on blood glucose levels may be related, at least in part, to an intestinal action of the drug on glucose transport. It remains to be determined whether the observed effect of aspirin on glucose absorption significantly alters glucose levels in patients with diabetes mellitus. 
This study was supported by the Kansas University Endowment Association (Grant 2550), General Research (Grants 2553 and 1542), and in part by Grant (RR-828) from the General Clinic Research Centers Program of the Division of Research Resources, National Institutes of Health.

\section{References}

Brandborg, L. L., Rubin, C. E., and Quinton, W. E. (1959). A multipurpose instrument for suction biopsy of the esophagus, stomach, small bowel and colon. Gastroenterology, 37, 1-16.

Brody, T. M. (1955). Uncoupling of oxidative phosphorylation as a mechanism of drug action. Pharmacological Reviews, 7, 335-363.

Cooper, H., Levitan, R., Fordtran, J. S., and Ingelfinger, F. J. (1966). A method for studying absorption of water and solute from the human small intestine. Gastroenterology, 50, 1-7.

Gilgore, S. G. (1960). The influence of salicylate on hyperglycemia. Diabetes, 9, 392-393.

Gould, B. J., and Smith, M. J. H. (1965). Inhibition of rat brain glutamate decarboxylase activity by salicylate in vitro. Journal of Pharmacy and Pharmacology, 17, 15-18.

Hahn, K. J., Krischkofski, D., Weber, E., and Morgenstern, E. (1975). Morphology of gastrointestinal effects of aspirin. Clinical Pharmacologv and Therapeutics, 17, 330-338.

Hecht, A., and Goldner, M. G. (1959). Reappraisal of the hypoglycemic action of acetylsalicylate. Metabolism, 8, 418-428.

Huggett, A. St. G., and Nixon, D. A. (1957). Use of glucose oxidase, peroxidase and o-dianisidine in determination of blood and urinary glucose. Lancet, 2, 368-370.

Kasbekar, D. K. (1973). Effects of salicylate and related compounds on gastric HCL secretion. American Journal of Physiology, 225, 521-527.

Kuo, Y. J., and Shanbour, L. L. (1976). Mechanism of action of aspirin on canine gastric mucosa. American Journal of Physiology, 230, 762-767.

Malawer, S. J., and Powell, D. W. (1967). An improved turbidimetric analysis of polyethylene glycol utilizing an emulsifier. Gastroenterology, 53, 250-256.

Morgan, C. R., Sorenson, R. L., and Lazarow, A. (1964). Studies of an inhibitor of the two antibody immunoassay system. Diabetes, 13, 1-5.

Pachman, L. M., Esterly, N. B., and Peterson, R. D. A. (1971). The effect of salicylate on the metabolism of normal and stimulated human lymphocytes in vitro. Journal of Clinical Investigation, 50, 226-230.

Reid, J., and Lightbody, T. D. (1959). The insulin equivalence of salicylate. British Medical Journal, 1, 897-900.

Smith, M. J. H., Meade, B. W., and Bornstein, J. (1952). The effect of salicylate on glucosuria, blood glucose and liver glycogen of the alloxan-diabetic rat. Biochemical Journal, 51, 18-20.

Snider, D. E., and Parker, C. W. (1975). Lower lymphocyte cyclic AMP levels after aspirin. New England Journal of Medicine, 292, 809-810.

Somogyi, M. (1945). Determination of blood sugar. Journal of Biological Chemistry, 160, 69-73.

Strehler, B. L., and Totter, J. K. (1954). Determination of ATP and related compounds: firefly luminescence and other methods. In Methods of Biochemical Analysis, vol. 1, pp. 341-356. Edited by D. Glick. Interscience Publishers: New York.

Trinder, P. (1954). Rapid determination of salicylate in biological fluids. Biochemical Journal, 57, 301-303.

Whalen, G. E., Harris, J. A., Geenen, J. E., and Soergel, K. H. (1966). Sodium and water absorption from the human small intestine. Gastroenterology, 51, 975-984.

Woodbury, D. M. (1970). Anagelsic-antipyretics, antiinflammatory agents and inhibitors of uric acid synthesis. In The Pharmacological Basis of Therapeutics, 4th edn, pp. 314-347. Edited by L. S. Goodman and A. Gilman. Macmillan: New York. 